\title{
DEMOGRAPHIC, CLINICAL AND MORPHOLOGICAL PROFILE OF MALIGNANT CUTANEOUS MELANOMA IN A NORTHEASTERN REGION OF BULGARIA
}

\author{
Lilyana Petkova, George St. Stoyanov \\ Department of General and Clinical Pathology, Forensic Medicine and Deontology, \\ Faculty of Medicine, Medical University of Varna
}

\begin{abstract}
INTRODUCTION: Melanoma is a malignant tumor with an unfavorable prognosis and an increasing incidence worldwide, with the most significant and the most common type being cutaneous melanoma. The epidemiological characteristics of cutaneous melanoma in Bulgaria are presented with low morbidity, moderate growth rate, and relatively low mortality, but with a rapid rate of increase in both sexes.

AIM: The aim of this study was to establish the clinical and morphological features of cutaneous melanoma in the Dobrich district and compare them to the European trends.

MATERIALS AND METHODS: A retrospective non-clinical approach was used in the selection of patients. The study included patients with histologically proven skin melanoma in the period 2016-2019. Data on patient sex, age at diagnosis, tumor location, and clinical stage of the disease were collected according to Clark and Breslow criteria.

RESULTS: The collected data show that the cutaneous melanoma in Dobrich district has a profile different from the average European one-all age groups are affected, except for 11- to 20-year-olds, and most cases occur in the age group of 61- to 70 -year-olds, with an average age of diagnosis 59.82 years. In regard to sex distribution, men are more common, and the most common localization is the lower limbs. Morphological features show an advanced nature of the disease with a diagnosis in the late clinical stages.

CONCLUSION: Our data show that the gender distribution of cases corresponds to the European average, but the age and clinical stage of the disease are significantly more advanced, indicating a delayed diagnosis and emphasizing the need to develop a campaign for the prevention and early diagnosis.
\end{abstract}

Keywords: cutaneous melanoma, demographics, morphology, clinical profile

Address for correspondence:

George St. Stoyanov

Faculty of Medicine

Medical University of Varna

55 Marin Drinov St

9002 Varna

e-mail:georgi.geesh@gmail.com

Received: August 19, 2021

Accepted: September 6, 2021

\section{INTRODUCTION}

Melanoma is a malignant tumor of melanocytic origin, unfavorable prognosis, and increasing frequency worldwide (1). The most significant and most frequent type of melanoma is cutaneous melanoma (2). It is a relatively rare malignancy, but it is the most common cause of death from malignant skin tumors, with increasing mortality. However, the mortality rate in Europe is relatively low and 
shows a trend of stabilization and reduction, mainly in Northwestern Europe (2-4). Mortality in Eastern Europe is the highest across the continent, however, it shows a trend of increased survival.

Cutaneous melanoma in Bulgaria presents with low morbidity, a moderate increase in incidence, relatively low mortality in men, and a medium-high one in women, but with a relatively rapid rate of increase in both sexes $(2,5-8)$.

The study of the features and trends in the prevalence of melanoma in the Bulgarian population, especially the demographic and morphological aspects of the disease, can provide a map to identify risk groups and lead to early diagnosis of cutaneous melanoma.

\section{AIM}

The aim of this study was to establish the clinical and morphological features of cutaneous melanoma in the Dobrich district and compare them to the European trends.

\section{MATERIALS AND METHODS \\ Ethical Approval}

All aspects of the study were carried out according to the ethical standards of the Declaration of Helsinki from 1975 and its seventh revision from 2013, the ethical norms and regulations of the Ministry of Education and Science, and the Ministry of Health of the Republic of Bulgaria. The protocol for approval by the Ethics Committee of the Medical University of Varna was as follows: №91/27.02.2020.

\section{Financing}

Part of the materials for this research were provided by project 19005/20 of the Science Fund at the Medical University of Varna.

\section{Selection of Patients}

A retrospective non-clinical approach was used in the selection of patients. The study included patients with histologically proven skin melanoma, initially diagnosed in Diagnostic and Consultative Center 2 Ltd., Dobrich, in the period 2016-2019. Data regarding the gender of patients, age at diagnosis, tumor location, and clinical stage of the disease (Clark and Breslow) were collected from the central database.

\section{Statistical Data Processing}

The collected data was analyzed and graphically represented using the built-in features of Microsoft Office 365 (Microsoft Corporation, Redmond, Washington, United States).

\section{RESULTS}

The study cohort included a total of 28 patients who met the set criteria, of which $57.14 \%(\mathrm{n}=16)$ were male and $42.86 \%(n=12)$ female (Fig. 1A). Cases were observed in all age groups, except for 11- to 20-yearolds, with the highest incidence being observed in the age group of 61 to 70 years, followed by these of 71 to 80 years and 51 to 60 years (Fig. 1B).

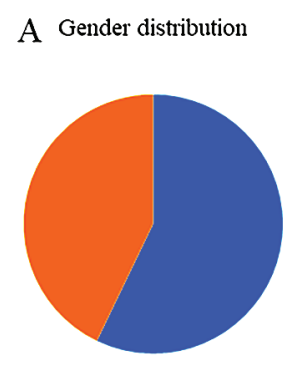

- Male $₫$ Female

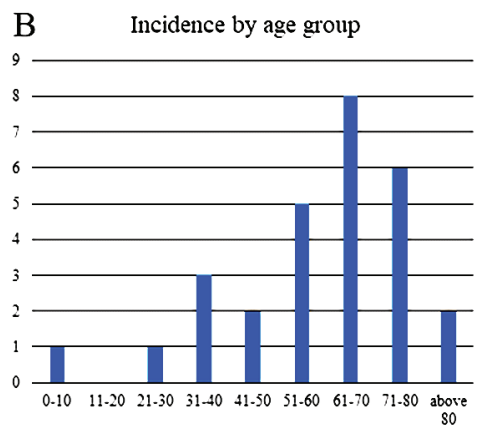

Fig. 1. Demographic characteristics of malignant melanoma-sex distribution (A) and age group distribution (B)

Clinical data on tumor location were available for 24 out of the 28 patients, with $25 \%$ of cases $(n=6)$ being located on the lower limbs, followed by the back and chest with $20.83 \%$ of cases $(n=5)$ each (Fig. 2).

Morphological examination of the excised tumor tissue showed that the majority of patients, regardless of gender and age, had tumors with advanced Clark and Breslow stages (Fig. 3), corresponding to a long period from their origin to the medical intervention.

\section{DISCUSSION}

According to the National Cancer Registry in the collections "Cancer Incidence in Bulgaria" for the previous four years-2012 to 2015, a total of 47 cases of cutaneous melanoma were diagnosed in Dobrich, with an annual incidence ranging between 7 and 16 cases per year and gender-age distribution 


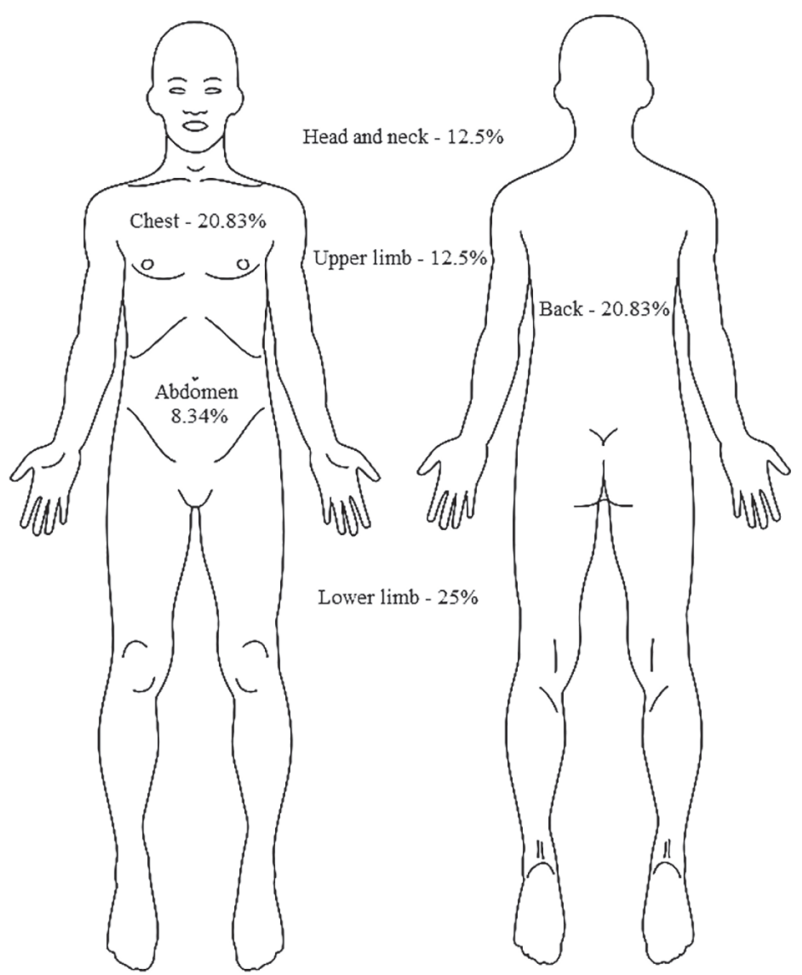

Fig. 2. Distribution of malignant melanoma by location
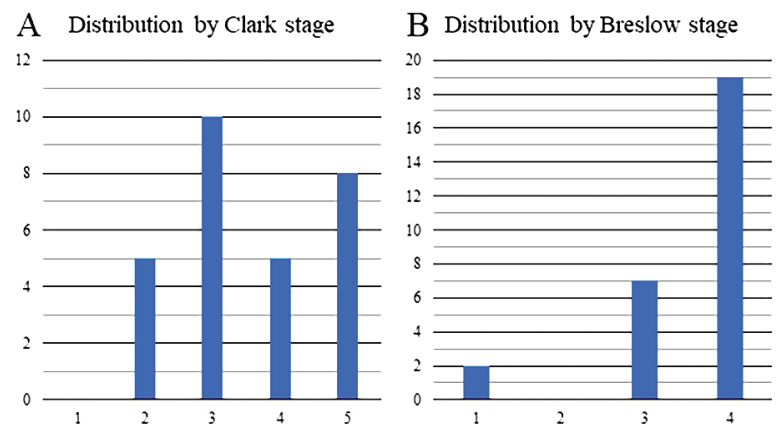

Fig. 3. Morphological characteristics of malignant melanoma according to the Clark scale $(A)$ and the Breslow scale (B)

corresponding to ours (9-11). Data on the incidence after 2015 have not yet been published but, based on the previous years and the corresponding age and gender distribution, most cases of melanoma in Dobrich district are included in our sample and make it representative.

According to Kobakova, the incidence of malignant melanoma in Bulgaria has increased at a moderate rate to 3.8 per 100,000 capita for males and 3.3 per 100,000 capita for females (12). This would characterize the annual incidence in Dobrich district of 3.26 cases for males and 2.98 for females by a trend of negative annual population growth of over 7 per thousand (13). As can be seen from the data of the National Cancer Registry and our statistics, however, the cases are significantly higher than the average morbidity for the country (9-11). Calculated by statistical means, the newly diagnosed cases should be 6 annually, with the registered ones being between 7 and 16 (12).

Probably one of the reasons for the higher incidence, when compared to the national average of cutaneous melanoma in the Dobrich region, is related to the agricultural nature of the livelihood of a large part of the population increasing the exposure to the main risk factor-UV radiation (14).

Our data show that malignant melanoma, as a nosological unit, is diagnosed in all age groups, apart from the age group of 11- to 20-year-olds in which no patients were registered. The most common location for the development of the disease is the lower limb. According to Chernev and Chokoeva, this location is associated with better prognosis when compared to the trunk, head, and neck areas (15).

Worthy of note is the fact that the disease is advanced, at an advanced clinical stage at the time of diagnosis, which limits the therapeutic response and inevitably reduces the survival rate for a disease with pronounced metastatic potential, including decades after removal of the primary lesion (16).

Bearing in mind the aforementioned data, it is necessary to develop a unified national system for prevention and early diagnosis, which includes dermatologists, surgeons, pathologists, and oncologists $(15,17)$. Informing the population about the main risk factors, especially in an agricultural region, is key to limiting exposure and seeking medical help in the early stages of the disease. This would improve survival and bring it in line with European trends, as well as inevitably increase the number of newly diagnosed cases in an earlier clinical phase, given the discovery of hidden cases for current statistics (2).

Additional evidence for the delayed diagnosis is evident from the data on gender distribution, which corresponds to the average European norms, whilst the age trend is lagging behind the European ones.

\section{Study Limitations}

The present study, although representative as per percentage of the total cases according to the na- 
tional databases of the previous years, does not present a complete analysis of all cases in the region. Further regional studies are required in all regions of the country to properly determine the incidence rates and underline the need for prevention programs in high-risk areas.

\section{CONCLUSION}

Malignant cutaneous melanoma is an aggressive malignancy with a poor clinical prognosis in its late clinical stages. Our data show that in the Dobrich district the gender distribution of cases corresponds to the European average, with the lower limb being the leading location of origin, but the age and clinical stage of the disease are significantly more advanced, indicating late diagnosis and emphasizing the need to develop a campaign for prevention and early diagnosis.

\section{REFERENCES}

1. Ali Z, Yousaf N, Larkin J. Melanoma epidemiology, biology and prognosis. EJC Suppl. 2013;11(2):81-91. doi: 10.1016/j.ejcsup.2013.07.012 .

2. Matthews NH, Li WQ, Qureshi AA, Weinstock MA, Cho E. Epidemiology of Melanoma. In: $\mathrm{Cu}-$ taneous Melanoma: Etiology and Therapy. Codon Publications; 2017. pp 3-22.

3. Forsea AM. Melanoma epidemiology and early detection in Europe: Diversity and disparities. Dermatol Pract Concept. 2020;10(3):e2020033. doi: 10.5826/dpc.1003a33.

4. de Vries E, Boniol M, Doré JF, Coebergh JW; EUROCARE working group. Lower incidence rates but thicker melanomas in Eastern Europe before 1992: a comparison with Western Europe. Eur J Cancer. 2004;40(7):1045-52. doi: 10.1016/j. ejca.2003.12.021.

5. Dimitrova N. Epidemiology of cutaneous melanoma - where is Bulgaria under the sun of Europe. In: MORE, Clinical management of cutaneous melanoma, basocellular carcinoma and Merkel-cell carcinoma. Art tracer; 2015. pp 11-21.

6. Barbaric J, Sekerija M, Agius D, Coza D, Dimitrova $\mathrm{N}$, Demetriou A, et al. Disparities in melanoma incidence and mortality in South-Eastern Europe: Increasing incidence and divergent mortality patterns. Is progress around the corner? Eur J Cancer. 2016 Mar;55:47-55. doi: 10.1016/j.ejca.2015.11.019.
7. Andreev VC. Diagnosis and treatment of malignant melanoma in Bulgaria. Br J Dermatol. 1968;80(3):149-52. doi: 10.1111/j.1365-2133.1968. tb12284.x.

8. Anchev N, Popov I, Ikonopisov RL. Epidemiology of Malignant Melanoma in Bulgaria. In: Structure and Control of the Melanocyte. Springer; 1966.

9. Valerianova Z, Dimitrova N, Tonev S, Vukov M, editors. Cancer Incidence in Bulgaria 2012. 23th ed. Sofia: Paradigma; 2014. (in Bulgarian).

10. Valerianova Z, Dimitrova N, Vukov M, Atanasov T, editors. Cancer Incidence in Bulgaria, 2013. 24 $4^{\text {th }}$ ed. Sofia: Paradigma; 2015. (in Bulgarian).

11. Valerianova Z, Atanasov T, Vukov M, editors. Cancer Incidence in Bulgaria, 2014 \& 2015. 25 ${ }^{\text {th }}$ ed. Sofia:Paradigma; 2017. (in Bulgarian).

12. Kobakova I. Epidemiology of cutaneous melanoma. Varna Med Forum. 2017; 6(1):69-74. doi: 10.14748/ vmf.v6i1.2066. (in Bulgarian).

13. National Institute of statistics [Internet]. Dobrich. (2020) [cited $20^{\text {th }}$ July 2020]. Available from: https:// www.nsi.bg/bg/content/11412/област-добрич

14. Rastrelli M, Tropea S, Rossi CR, Alaibac M. Melanoma: epidemiology, risk factors, pathogenesis, diagnosis and classification. In Vivo. 2014;28(6):1005-11.

15. Chernev G, Chokoeva A. Malignant Melanoma. Sofia: St. Kliment Ohridski University Publishing; 2015. (in Bulgarian).

16. Gershenwald JE, Scolyer RA, Hess KR, Sondak VK, Long GV, Ross MI, et al. Melanoma staging: Evidence-based changes in the American Joint Committee on Cancer eighth edition cancer staging manual. CA Cancer J Clin. 2017;67(6):472-92. doi: 10.3322/caac. 21409 .

17. Chokoeva AA, Tchernev G, Philipov S, Cardoso JC, Zanardelli M, Lotti T. Wrong melanoma thickness measurement: check it or leave it? Int J Immunopathol Pharmacol. 2014;27(4):639-44. doi: $10.1177 / 039463201402700421$. 